\title{
Assessing Mobile Touch Interfaces for Tetraplegics
}

\author{
Tiago Guerreiro, Hugo Nicolau, Joaquim Jorge, Daniel Gonçalves \\ IST / Technical University of Lisbon / INESC-ID \\ R. Alves Redol, 9, 1000-029 Lisbon, Portugal \\ $+351214233565$
}

\{tjvg, hman, jaj\}@vimmi.inesc-id.pt, daniel.goncalves@inesc-id.pt

\begin{abstract}
Mobile touch-screen interfaces and tetraplegic people have a controversial connection. While users with residual capacities in their upper extremities could benefit immensely from a device which does not require strength to operate, the precision needed to effectively select a target bars these people access to countless communication, leisure and productivity opportunities. Insightful projects attempted to bridge this gap via either special hardware or particular interface tweaks. Still, we need further insight into the challenges and the frontiers separating failure from success for such applications to take hold. This paper discusses an evaluation conducted with 15 tetraplegic people to learn the limits to their performance within a comprehensive set of interaction methods. We then present the results concerning a particular interaction technique: Tapping. Results show that performance varies across different areas of the screen whose distribution changes with target size.
\end{abstract}

\section{Categories and Subject Descriptors}

D.3.3 [Information Interfaces and Presentation]: User Interfaces - Input devices and strategies, User-centered design.

\section{General Terms}

Performance, Design, Experimentation, Human Factors.

\section{Keywords}

Touch-Screen, Tetraplegic, Interaction, Tapping, Evaluation, Mobile device.

\section{INTRODUCTION}

Touch-screen interfaces are increasingly replacing traditional keypads in mobile phones. These stylish devices provide a more natural and engaging user experience and promise to be more effective and efficient than their predecessors. However, and besides one being able to argue that this goal has not yet been achieved, what is also true is that they are also highly physically demanding. Due to the absence of relief, touch-screens make it harder for people to accurately select targets. This problem occurs with everyone, particularly when situationally impaired [8] but also with motor disabled users who suffer from lack of precision when moving their upper extremities. On the other hand, touch screens are less demanding than keypads, regarding the strength required to select a target. This presents good opportunities to motor disabled people, particularly those who lack both strength and control on their upper limbs [3]. Yet, operating these screens is still demanding for even the most capable users, let alone disabled people, such as tetraplegics.

There have been successful efforts to improve access to mobile touch screen interfaces by motor disabled people. Examples

Copyright is held by the author/owner(s). MobileHCI 2010 September 7 - 10, 2010, Lisboa, Portugal. ACM 978-1-60558-835-3.

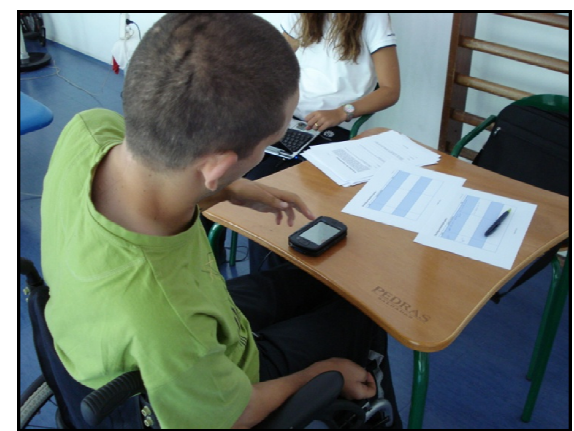

Figure 1 - Motor-impaired person interacting with a mobile touch-screen during evaluation

include EdgeWrite [9], a stylus-based approach that uses edges and corners of a reduced touch-screen to enable text-entry tasks on a PDA. Similarly, Barrier Pointing [2] enables motor impaired users to operate devices by stroking towards the screen barriers (edges and corners).

However, it is still very difficult to design better interfaces for severely disabled people who could benefit immensely from proper access to mobile devices. To overcome this, we have performed extensive evaluations with tetraplegic people (Figure 1). These included testing different methods such as tapping, gesturing or crossing targets, and exploring the screen characteristics in search of guidelines to a better user experience (e.g., are corners or edges good target positions?).

This paper presents two main contributions. First, we provide an evaluation script which contemplates a comprehensive set of interaction primitives and their instantiations (Figure 2). Second, we analyze a particular interaction technique: Tapping a target. Our attention focused on target sizes and screen locations that are commonly associated with either improved or reduced performance.

\section{TOWARDS ACCESSIBLE TOUCH INTERFACES}

Recently, there have been significant efforts applied to make mobile devices accessible to disabled people [2, 3, 9]. In particular, touch screen devices pose both challenges and opportunities for researchers. Indeed, while they may enable a less physically demanding experience, they are less explored and understood than traditional keypad-based appliances.

Thanks to developments in ICT, motor disabled users are now able to use hardware and software-based interfaces that promise to improve their relation with technology. The aforementioned projects attest to this. However, we still need to understand how motor-impaired people use these devices, what tasks they can carry out, and which device features can be adapted (and how) to 


\section{,}
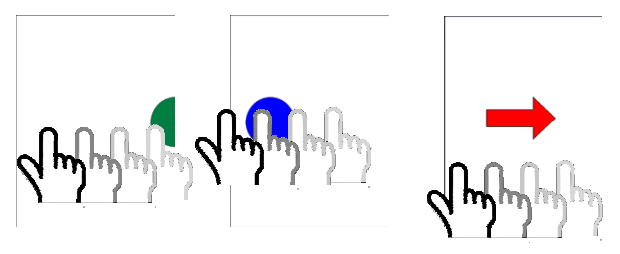

Figure 2 - Tapping, Exiting, Crossing, Gesturing maximize their effectiveness. We need to take a step back from current research and ask whether for motor-impaired users: Is tapping on a screen easier than crossing a target or performing a directional gesture? Are there any preferred screen areas, be those corners, edges or sides? These are examples of questions we intended to answer with the studies presented henceforth.

To this end, we studied tetraplegic people interacting with touchscreen mobile devices to assess which primitives and characteristics better suit their general condition and individual characteristics. In this paper, we present the study and how it was conducted and report on a particular subset of the results. We considered two basic interaction methods: tapping the screen or performing a gesture. Users could tap the screen in three different areas: edges, corners or the remaining surface. When performing a gesture, the user can cross a target (and therefore making a selection), acknowledge a selection by exiting the screen via a specific target, or just use directional gestures. Those gestures can be performed in the middle of the screen or using the edges as guidelines. We present user studies regarding different interaction methods (tapping, crossing, gesturing, and exiting Figure 2), different target sizes (7, 12 and $17 \mathrm{~mm})$ and target positions (Figure 3). The target sizes were selected taking in consideration the experiments reported for able bodied users (minimum $3 \mathrm{~mm}$ and suggested between 9.2 and $9.6 \mathrm{~mm}$ ) [5, 6, 7].

\subsection{Participants}

We recruited 15 tetraplegic participants (two female) averaging 42 years old from a physical rehabilitation center. Figure 4 presents information regarding user profile, lesion level and a preliminary capability (grasp) assessment [1]. This functional evaluation was performed prior to the experience itself and aims at more concrete capability identification (in opposite to lesion level).

All users possessed a mobile phone, but most never experienced with a touch-screen mobile phone before and found some
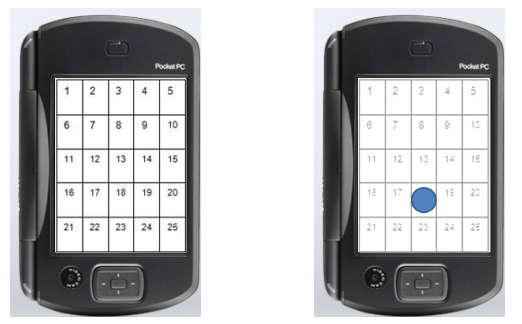

Figure 3 - Qtek 9000. (Left) Screen areas (Right)

difficulties understanding its operation. As a baseline, 18 ablebodied participants (five female) were also evaluated.

\subsection{Apparatus}

The studies were performed using a QTEK 9000 PDA (Figure 3) running Windows Mobile 5.0. It sports a relatively large screen (73x55 mm) 640x480 pixels wide. The evaluation software was developed in C\#, using the .NET Compact Framework 3.5 and Windows Mobile 5.0 SDK. The evaluation was video recorded and all the interactions were logged for posterior analysis.

\subsection{Procedure}

Each user was asked to perform target selections in all screen areas as shown in Figure 3 (tapping, crossing), all directions (gesturing) including repeated directions with edge-support (e.g., north somewhere on the screen and north using the edge as a guideline). Exiting, where the user performs a gesture towards the target leaving the screen, a technique between crossing and gesturing, had targets positioned at a corner or an edge which had to be crossed into. We used a within-subject design. This meant all users were asked to perform all target acquisitions using three different target sizes (7, 12 and $17 \mathrm{~mm}$ ). Each user tested each interaction method and sizes separately. We selected tests in a random order to avoid bias associated with experience. In each method-size experience set, target positions were also prompted randomly to counteract order effects. Each acquisition was restricted to one attempt. The next target appeared following a two-second delay after each action. Users were not informed on whether a task was successful. However, they received feedback that an action was performed. All acquisitions were stylus-free. However, users were free to issue selections with any part of their fingers (any finger). Each user performed 142 selections, totaling 2130 across all 15 disabled and 2556 for able-bodied subjects, respectively.

\begin{tabular}{|c|c|c|c|c|c|c|c|c|c|c|c|c|}
\hline$P$ & Age & Sex & Lesion Level & Lesion Type & Tip & Palmar & Two-finger & Lateral & Cylindrical & Tee & Spherical & Press \\
\hline 1 & 28 & $M$ & C4-C5 & Incomplete & Unable & Unable & Poor & Poor & Unable & Unable & Unable & Good \\
\hline 2 & 28 & $M$ & C5 & Complete & Unable & Unable & Good & Good & Fair & Poor & Poor & Fair \\
\hline 3 & 29 & $F$ & C5-C6 & Complete & Unable & Unable & Good & Good & Good & Good & Good & Good \\
\hline 4 & 28 & $F$ & C5 & Incomplete & Unable & Good & Good & Good & Good & Good & Good & Good \\
\hline 5 & 61 & $M$ & $C 5$ & Incomplete & Good & Good & Good & Good & Good & Good & Good & Good \\
\hline 6 & 30 & $M$ & $C 4-C 5$ & Complete & Unable & Unable & Poor & Poor & Unable & Unable & Unable & Poor \\
\hline 7 & 34 & $M$ & C4 & Incomplete & Good & Unable & Good & Poor & Fair & Unable & Good & Good \\
\hline 8 & 40 & $M$ & $C 6-C 7$ & Complete & Unable & Good & Good & Good & Good & Good & Good & Good \\
\hline 9 & 61 & $M$ & $C 4-C 5$ & Complete & Good & Good & Good & Good & Good & Good & Good & Good \\
\hline 10 & 42 & $M$ & $C 5-C 6$ & Complete & Unable & Poor & Good & Good & Good & Good & Good & Good \\
\hline 11 & 58 & $M$ & $C 5-C 6$ & Incomplete & Unable & Good & Good & Good & Good & Good & Good & Good \\
\hline 12 & 58 & $M$ & $C 4-C 5$ & Complete & Unable & Unable & Unable & Poor & Unable & Unable & Unable & Fair \\
\hline 13 & 44 & $M$ & C5-C6 & Incomplete & Unable & Good & Good & Good & Fair & Fair & Poor & Good \\
\hline 14 & 64 & $M$ & C5 & Incomplete & Unable & Poor & Fair & Fair & Fair & Fair & Good & Good \\
\hline 15 & 27 & $M$ & C4-C5 & Complete & Unable & Unable & Unable & Poor & Unable & Unable & Unable & Good \\
\hline
\end{tabular}

Figure 4 - User profile and grasp assessment results 

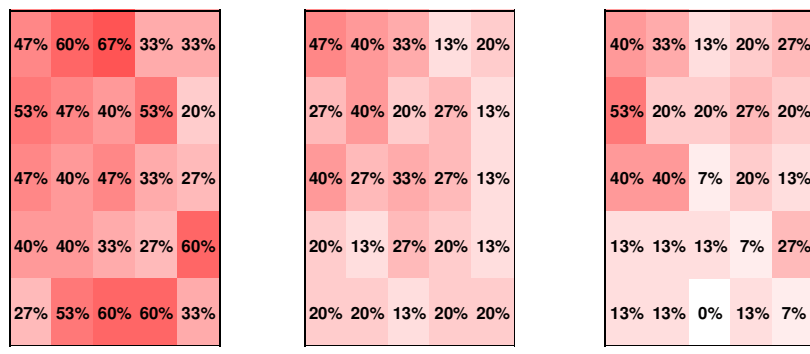

Figure 5 - Error rate heat map for each size: $7 \mathrm{~mm}$ (left), $12 \mathrm{~mm}$ (center), and $17 \mathrm{~mm}$ (right). The heat map relates to target center even though targets have different sizes.

All sessions were performed in a comfortable and quiet place (their homes or rehabilitation center facilities) always with the users sitting on their chairs either with a table or armrest in front of them.

This evaluation aims to answer several research questions, regarding motor-impaired people:

1. What are the best screen areas for users to tap?

2. Are people more accurate when tapping targets on screen edges?

3. Are targets placed on screen corners more accurately tapped?

4. Is it easier to perform gestures on the edges than on the middle of the screen?

5. What is the best size for both target acquisition and accuracy, regarding different screen locations?

6. What is the best interaction method for disabled people?

7. What is the best method-size-area combination?

This paper focuses on a particular method and thus, on a subset of the described experience: Tapping. The next section presents the goals, hypothesis and results obtained exploring this method with tetraplegic subjects.

\section{HOW TETRATPLEGIC USERS TAP?}

Tapping a screen is still the most common interaction technique performed with a touch screen mobile phone. However, it is also one of the most demanding, as it requires precisely acquiring a particular location on the screen. Moreover, incorrect selections originate errors, burdening users with error recovery mechanisms, and requiring new attempts to achieve accurate selection. These problems are more troublesome for tetraplegic people as, not only can they select targets at a lower precision, but they also have a harder time recovering from errors. To design suitable interfaces

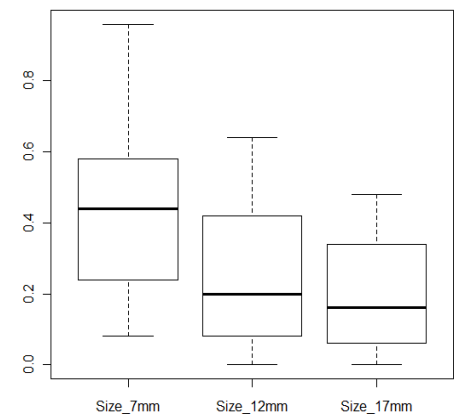

Figure 6 - Task Error rates vs. Target Size for tetraplegic users we need to step back from common practice and look at challenges anew.

\subsection{Goals and Hypothesis}

Our goal is to understand how tetraplegic people select targets on a screen by touching it. We call this action Tapping. We aim to comprehend what are the best interface settings for this user population in applying the technique. We also would like to learn if there are any particular attributes that affect their performance. Thus, this experiment considers first the users' characteristics, and second, both target size and position. The only dependent variable considered is Task Errors as no time limit was imposed.

\subsection{Results}

Figure 5 presents Task Error rates for each screen area, averaged over all 15 users. We define Task Errors as an incorrect tap (touching another screen area). This variable fits a normal distribution (Shapiro-Wilk, W=0.9, p >.01). We applied further analysis using ANOVA. When required to verify where significant differences occur, we performed multiple comparisons using Tukey's post-hoc test.

\subsubsection{User Profile}

We can classify a person's tetraplegy by the vertebrae which were compromised. However this is not a reliable characterization of their physical capability. Indeed, the type of lesion (complete or incomplete), the rehabilitation process and other uncontrolled variables make this relation not very reliable.

Nevertheless, functional abilities, as the arm reach or grasp precision, seem more likely to influence the ability to effectively control a mobile device. To this end, we have performed the functional evaluations depicted in [1] (results are presented in Figure 4) and tried to correlate the results with user's effectiveness. However, we found no significant correlation either between the principal components of the evaluation set or with any particular user characteristics and Task Errors. This indicates further studies are needed to find functional characteristics that reliably predict user performance when interacting with mobile touch screens.

\subsubsection{Target Size}

Previous studies derived recommendations on target sizes and position for mobile touch screen interfaces [5,6,7]. Indeed, this is true for able-bodied users in static environments and also for situationally impaired subjects $[8,4]$. However, to our knowledge studies of motor-challenged users have yet to be carried out, particularly for tetraplegics with residual arm movement.

Figure 6 presents Task Error rate results for each target size. There was a significant effect of size on Task Errors $\left(\mathrm{F}_{1,14}=25.10\right.$, $\mathrm{p}<.001$ ). A multiple comparisons post-hoc test (Tukey's) found significant differences between small and medium sizes and also between small and large sizes. These results indicate $12 \mathrm{~mm}$ as an approximate suitable value for targets to be acquired by motor impaired users. Further studies will likely yield the best relation between size, target distribution on screen and user performance.

\subsubsection{Corner Targets}

The lack of precision in tetraplegic users' movements suggests that additional supports or aids need to be provided to enhance their performance. Regular mobile touch screens usually present borders, hence creating corners and edges with a noticeable relief (the evaluation was performed with one of these devices. Not all touch screen devices guarantee this property, e.g., iPhone). We 


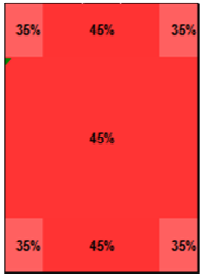

a)

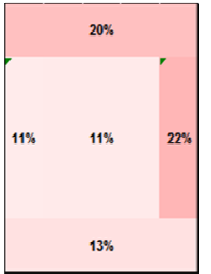

b)

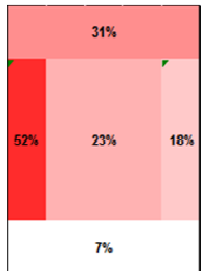

c)

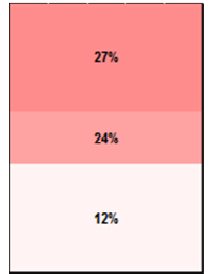

d)
Figure 7 - Task Error rates: a) Corners; Edges with b) lefthanded users and c) right-handed users; d) Vertical

tested whether tapping a target in the corner of the screen (4 corners) is indeed easier than tapping a target in any other position. We found no significant effect of target position (corner or center) on Task Errors (large and medium sizes). A minor effect $\left(\mathrm{F}_{1,14}=3.1, \mathrm{p}<.1\right)$ was found on the smallest size (less errors in the corners) (Figure 7.a). Finally, we found no significant difference between the specific corners and no interaction between corners and which arm was used for any of the tested target sizes.

\subsubsection{Edge Targets}

Considering edges, we could find no significant difference on Task Errors between tapping a target, regardless of its size, whether placed on an edge or not. However, considering each individual edge, we found a significant difference between the which edge was chosen $\left(\mathrm{F}_{1,3}=2.9, \mathrm{p}<.05\right)$ for the largest size. We also found a minor interaction between the preferred arm used and the error rate on a specific edge for medium size $\left(\mathrm{F}_{1,3}=2.3, \mathrm{p}<.1\right)$, and a significant interaction between which arm was used and a specific edge for the largest size $\left(\mathrm{F}_{1,3}=3.2, \mathrm{p}<.05\right)$ (Figure 7.b and 7.c).

\subsubsection{Screen areas}

Even though mobile touch screens are relatively small, we believe that users have different success rates depending on the position of the target and physical abilities such as arm reach. To validate this belief we tested the difference between Error rates in different areas of the screen, both from horizontal (left, center, right ) and vertical (top, center, down) perspectives.

Horizontally, we found target positions had no significant effect on Task Errors. However, for the vertical case, we found a significant difference in the largest size $\left(\mathrm{F}_{1,2}=3.9, \mathrm{p}<.05\right)$ (Figure 7.d) and a minor effect in the medium size $\left(\mathrm{F}_{1,2}=3.3, \mathrm{p}<.1\right)$. We found no interaction with the arm used in either test.

\subsection{Design Implications}

The most relevant implication suggested by these evaluations is that motor disabled users can operate devices with different success rates depending on screen position. While other people can adjust the device to their convenience, severely motor challenged users have less freedom to interact with the device.

As for size, the minimum target for able-bodied people $(3 \mathrm{~mm})$ does not work for tetraplegics. Indeed for tetraplegics, results showed that error rates start to level off above $12 \mathrm{~mm}$, which indicates that a good target size would be around this value.

Concerning target positioning, the major implication is that significant effects are mostly observed at larger sizes.
Furthermore, it is easier to select targets at the bottom of the screen. Targets next to the preferred arm are also easier to select. Indeed, visual inspection suggests that for a right handed-user, the greater the distance from the bottom right corner (closer to the user's support), the higher the error rate. One limitation of this evaluation was the small number of left-handed users, which limits our conclusions on target locations on the screen (a particular good corner or good edge) most suitable for either hand.

\section{CONCLUSIONS}

We undertook an extensive evaluation with 15 tetraplegic users and a wide set of mobile touch screen interaction methods and primitives. Thus, we contribute both an evaluation script and results for a particular method: Tapping. These show that tetraplegic users are likely to benefit from a better understanding of their abilities and challenges, particularly considering target size and position.

In the future, we will analyze more interaction methods and the relationships between them. Moreover, we will analyze the differences between tetraplegics and able-bodied people in greater depth, in order to understand where they diverge and identify where extra attention is required when designing interfaces.

\section{ACKNOWLEDGMENTS}

The authors would like to thank all the users that participated in the studies. This work was supported by FCT (INESC-ID multiannual funding) through the PIDDAC Program funds. Hugo Nicolau and Tiago Guerreiro were supported by the Portuguese Foundation for Science and Technology, grants SFRH/BD/46748/2008 and SFRH/BD/28110/2006, respectively.

\section{REFERENCES}

[1] Cook, A. and Hussey, S. Assistive Technologies - Principles and Practice. Mosby, 2002.

[2] Froehlich, J.,et al. Barrier Pointing: Using Physical Edges to Assist Target Acquisition on Mobile Device Touch Screens. Proc. of ACM ASSETS (2007), 19-26.

[3] Myers, B. A., et al.. Using Handhelds to Help People with Motor Impairments. Proc. of ACM ASSETS (2002), 89-96.

[4] Mizobuchi, S., Chignell, M., and Newton, D. Mobile text entry: relationship between walking speed and text input task difficulty. Proc. MobileHCI (2005), 122-128.

[5] Parhi, P., et al. Target Size Study for One-Handed Thumb Use on Small Touchscreen Devices. Proc. MobileHCI (2006).

[6] Park, Y. S., et al. Touch Key Design for Target Selection on a Mobile Phone. Proc. 10th MobileHCI (2008), 423-426.

[7] Perry, K. B. et al.. Evaluating One Handed Thumb Tapping on Mobile Touchscreen Devices. Proc . GI (2008), 57-64.

[8] Sears, A., et al. When Computers Fade. Pervasive Computing and Situationally-Induced Impairments and Disabilities, pp. 1298-1302. Lawrence Erlbaum Associates, 2003.

[9] Wobbrock, J. O., Myers, B. A., and Kembel, J. A EdgeWrite: A Stylus-Based Text Entry Method Designed for High Accuracy and Stability of Motion. UIST (2003), 61-70. 